\title{
PROCESOS, DIMENSIONES Y HERRAMIENTAS PARA LA GESTIÓN DEL CONOCIMIENTO EN EL MANTENIMIENTO INDUSTRIAL
}

\section{PROCESSES, DIMENSIONS AND TOOLS FOR THE MANAGEMENT OF KNOWLEDGE IN INDUSTRIAL MAINTENANCE}

\author{
F. Javier Cárcel Carrasco* E-mail: fracarc1@csa.upv.es \\ Manuel Rodriguez Méndez \\ *Universidad Politécnica de Valencia, Camino de Vera \\ ${ }^{* *}$ Aseypro S.L. Director de estudios industriales
}

\begin{abstract}
Resumen: La gestión del conocimiento, implantada en algunas áreas de la empresa, es normalmente olvidada en las propias organizaciones de mantenimiento que sin embargo, es una de las actividades donde se requiere un profundo conocimiento de los activos físicos de la empresa, y que influye en la propia fiabilidad de los procesos de explotación, la productividad de la empresa y es donde se encuentra mayor nivel de conocimiento tácito basado en las propia experiencia de los operarios. En este artículo se hace una revisión de la literatura, marcando los procesos, dimensiones y herramientas que pueden ser utilizadas para la gestión del conocimiento en el ámbito del mantenimiento industrial.
\end{abstract}

Palabras-clave: Mantenimiento industrial. Factor humano. Gestión del conocimiento.

Abstract: Knowledge management, implanted in some areas of the company, is usually forgotten in the own service organizations is one of the activities requiring a deep knowledge of the physical assets of the company, that influences the own reliability of the processes of exploitation, the productivity of the company and is where higher level of tacit knowledge based on the experience of the operators. This article is a review of the literature, marking processes, dimensions and tools that can be used for the management of knowledge in the field of industrial maintenance.

Keywords: Industrial maintenance. Human Factor. knowledge management.

\section{INTRODUCCIÓN}

Todas las perspectivas de la gestión del conocimiento, basándose en unos principios y unas prácticas, en muy pocas ocasiones son adscritas a la parte donde se almacena mayor nivel de conocimiento tácito dentro de las empresas industriales y de servicios, como pueden ser los aspectos tácticos de mantenimiento, montaje y proyectos, y actividades en general de difícil documentación, que normalmente se basan en gran parte en la experiencia de los empleados adquirida con los años, de difícil captación y aún más difícil transferencia. 
Aunque en numerosas ocasiones la gestión del conocimiento es infrautilizada y desplegada ineficientemente (ORDÓÑEZ, 1999,2001), se puede definir la gestión del conocimiento como "las estructuras, sistemas e interacciones integradas conscientemente y diseñadas para permitir la gestión del conjunto de conocimiento y habilidades de la empresa" (TIEMESSEN et al., 1997), convirtiéndose en un recurso de importancia estratégica fundamental (BUENO, 1999, 2002; HEDLUND, 1994; NONAKA Y TAKEUCHI, 1995; VENTURA, 1996; WERNERFELT, 1984).

La dimensión epistemológica del conocimiento distingue entre conocimiento tácito y conocimiento explícito (POLANYI, 1966). El conocimiento tácito es aquel que se adquiere a través de la experiencia. El conocimiento explícito o codificado (POLANYI, 1966) es aquel transmisible mediante el lenguaje formal y sistemático, y puede adoptar la forma de programas informáticos, patentes, diagramas o similares (HEDLUND, 1994). El conocimiento tácito no debe ser considerado independiente del conocimiento explícito, pues hay una dimensión tácita en todas las formas de conocimiento (POLANYI, 1966). En la Tabla 1 se recogen las principales diferencias entre ambos tipos de conocimiento:

Tabla 1 - Tipos de conocimiento

\begin{tabular}{|c|c|c|c|}
\hline \multirow{5}{*}{ 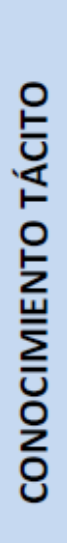 } & \multicolumn{2}{|c|}{ CARACTERISTICAS DEL TIPO DE CONOCIMIENTO } & \multirow{5}{*}{  } \\
\hline & $\begin{array}{l}\text { Conocimiento a través de la } \\
\text { experiencia. (Cuerpo) }\end{array}$ & $\begin{array}{l}\text { Conocimiento a través de } \\
\text { la racionalidad. (Mente) }\end{array}$ & \\
\hline & Conocimiento simultáneo & $\begin{array}{l}\text { Conocimiento secuencial } \\
\text { (en el acto) }\end{array}$ & \\
\hline & $\begin{array}{l}\text { Conocimiento analógico } \\
\text { (basado en la práctica) }\end{array}$ & $\begin{array}{l}\text { Conocimiento digital } \\
\text { (basado en la teoría) }\end{array}$ & \\
\hline & Subjetivo & Objetivo & \\
\hline
\end{tabular}

Fuente: Elaboración propia a partir de (NONAKA Y TAKEUCHI ,1995).

Es por ello fundamental para establecer proyectos de gestión de conocimiento en el ámbito de las organizaciones de mantenimiento industrial, definir cuáles son los procesos, dimensiones y herramientas que pueden ser utilizadas para la gestión del conocimiento en esta área estratégica. En este artículo se hace una revisión de la literatura sobre estos puntos, para que de una manera Revista Produção Online, Florianópolis, SC, v.14, n. 2, p. 720-743, abr./jun. 2014. 
introductoria se puedan tomar decisiones de la manera de iniciar un proyecto de este tipo.

\section{METODOLOGÍA}

Para la realización de este estudio se ha realizado una revisión bibliográfica con un alcance limitado al conocimiento de los procesos, dimensiones y herramientas para la gestión del conocimiento en su aplicación a la ingeniería del mantenimiento industrial. La estrategia de búsqueda bibliográfica y criterios de selección de los artículos ha sido en base a estructurar la información y estudios genéricos sobre la gestión del conocimiento que influyan en el desempeño del personal implicado en la ingeniería del mantenimiento industrial. Se han utilizado para la búsqueda de la información las bases de datos de la web of science (WOK), accesible vía internet desde las plataformas de investigación de la Universidad Politécnica de Valencia (España).

Teniendo en cuenta la temática, se ha estructurado el artículo en dos fases fundamentales: Una primera fase introductoria para describir los procesos y dimensiones que implica la gestión del conocimiento, En una segunda fase se analizan las herramientas básicas que pueden ser utilizadas para la adecuada gestión del conocimiento, y que pueden ser utilizadas en la ingeniería del mantenimiento industrial.

Por ello este estudio pretende ofrecer una base introductoria a los estudiosos de la ingeniería del mantenimiento industrial sobre la transcendencia que puede suponer sobre estas organizaciones y el personal afectado, para marcar la incidencia que puede producir la adecuada gestión del conocimiento.

\section{PROCESOS Y DIMENSIONES EN LA GESTIÓN DEL CONOCIMIENTO.}

Barragán analiza las taxonomías de modelos de gestión del conocimiento en donde se pueden encontrar puntos en común que permiten resumirlas y reagruparlas para poder homogenizar los criterios en áreas donde el estudio y desarrollo de la gestión del conocimiento han tenido un desenvolvimiento 
importante; entre estos criterios destacan aspectos teóricos, conceptuales, filosóficos, técnicos, científicos, cognitivos, de capital intelectual, sociales y de trabajo de la gestión del conocimiento como se describen en la tabla 2 (Barragán, 2009).

Tabla 2- Resumen de los principales criterios de clasificación encontrados en la literatura

\begin{tabular}{|c|c|}
\hline Criterios & Principales características \\
\hline $\begin{array}{l}\text { Conceptuales, teóricos, y } \\
\text { filosóficos. }\end{array}$ & $\begin{array}{l}\text { Modelos cuya principal característica consiste en enriquecer el } \\
\text { estudio de la gestión del conocimiento desde un enfoque teórico y } \\
\text { conceptual a partir del estudio de la epistemología y temas } \\
\text { relacionados con el conocimiento, lo que permite ahondar sobre el } \\
\text { entendimiento de este tipo de modelos. }\end{array}$ \\
\hline $\begin{array}{l}\text { Cognoscitivos y de capital } \\
\text { intelectual. }\end{array}$ & $\begin{array}{l}\text { Este tipo de modelos generalmente son desarrollados dentro de } \\
\text { organizaciones e industrias que buscan hacer un uso intensivo del } \\
\text { uso y aplicación del conocimiento con la finalidad de generar valor } \\
\text { para sus productos y procesos; así como también para la búsqueda } \\
\text { de soluciones a distintos problemas. }\end{array}$ \\
\hline Sociales y de trabajo & $\begin{array}{l}\text { En este rubro la principal característica que distingue a los } \\
\text { modelos, es el estudio de la socialización del conocimiento entre } \\
\text { distintos tipos de actores o grupos de trabajo con la finalidad de } \\
\text { entender y optimizar los mecanismos de uso y transferencia del } \\
\text { conocimiento para promover el beneficio social y/o grupal. }\end{array}$ \\
\hline Técnicos y científicos. & $\begin{array}{l}\text { Los modelos técnicos y científicos son aquellos que en una parte de } \\
\text { este tipo de clasificaciones se incluyen modelos que logran } \\
\text { incorporar el uso de las TIC para mejorar el uso y aplicación del } \\
\text { conocimiento. Pero por otra parte dentro de esta categoría se } \\
\text { incluyen también modelos que pretenden optimizar la gestión de la } \\
\text { investigación y desarrollo tecnológico que se lleva a cabo dentro de } \\
\text { una organización. }\end{array}$ \\
\hline
\end{tabular}

Fuente: (BARRAGÁN, 2009)

Las dimensiones en la gestión del conocimiento según los estudios empíricos y teóricos (TARÍ et al., 2009), que están relacionados directamente con el control de calidad, se pueden enumerar en las siguientes:

- Creación de conocimiento (aprendizaje organizativo): adquisición de información, diseminación de la información e interpretación compartida.

- Transferencia y almacenamiento de conocimiento (conocimiento organizativo): almacenar conocimiento y transferencia de conocimiento.

- Aplicación y uso del conocimiento (organización de aprendizaje): trabajo en equipo, promover el diálogo, establecer sistemas para capturar y compartir el aprendizaje, relación entre distintos departamentos o áreas funcionales y compromiso con el aprendizaje.

En las tablas 3, 4 y 5 se puede ver el resumen de los diferentes estudios con sus dimensiones teóricas a juicio de diversos autores. 
Tabla 3 - Dimensiones teóricas según la literatura.

\begin{tabular}{|c|c|}
\hline Estudios & Dimensiones teóricas \\
\hline \multicolumn{2}{|c|}{ Creacion (aprondizaje organizativo) } \\
\hline 1. Slater y Narver (1995) & $\begin{array}{l}\text { Adquisición de información, diseminación de la información, interpretación } \\
\text { compartida. }\end{array}$ \\
\hline 2. Crossan ot al. (1999) & Intuición, interpretación, integración, instimcionalización. \\
\hline $\begin{array}{l}\text { 3. Benavides y Escribá } \\
(2001)\end{array}$ & Trabajo en equipo, relaciones organizativas. \\
\hline 4. Escribà y Roig (2002) & Equipos de trabajo. \\
\hline 5. Marquardt (2002) & $\begin{array}{l}\text { Aprendizaje dinámico, transferencia de la organización, empowerment, } \\
\text { dirección de conocimieatos y aumento de la tecnologia. }\end{array}$ \\
\hline 6. Chiva y Camisón (2003) & $\begin{array}{l}\text { Experimentación, nuevas ideas, mejora continua, recompensas, apertura al } \\
\text { cambio, observación, apertura e interacción con el entomo, aceptación del } \\
\text { error y riesgo, heterogeneidad, diversidad, diálogo, comumicación y } \\
\text { construcción social, formación continua, delegación y participación, trabajo } \\
\text { en equipo, importancia del grupo, espintu colectivo, colaboración, } \\
\text { trabajadores con deseos de aprender, liderazgo comprometido, estructura } \\
\text { organizativa y directiva poco jerárquica y flexible, conocimiento de } \\
\text { objetivos y estrategias organizativas, accesibilidad e la información, sentido } \\
\text { del bumor, improvisación y creatividad. }\end{array}$ \\
\hline 7. Andreu et al. (2005) & $\begin{array}{l}\text { Compromiso con el aprendizaje, visión compartida y mentalidad } \\
\text { aperturista. }\end{array}$ \\
\hline 8. Chao et al. (2007) & $\begin{array}{l}\text { Aprendizaje de explotación, aprendizaje de exploración, conocimiento } \\
\text { tácito y conocimiento explicito. }\end{array}$ \\
\hline 9. Tippins y Sohi (2003) & $\begin{array}{l}\text { Adquisición de información, diseminación de la información, interpretación } \\
\text { compartida, Memoria declarativa y Memoria procesual }\end{array}$ \\
\hline \multicolumn{2}{|c|}{ Transforencia y almacenamionto (conocimiento organizativo) } \\
\hline 10. Guadamillas (2001) & Crear, almacenar, distribuir, aplicar. \\
\hline 11. Linderman et al (2004) & Socialización, exteriorización, combinación, interiorización \\
\hline \multicolumn{2}{|c|}{ Aplicación y uso (organización de aprendizaje) } \\
\hline 12. Garvin (1993) & $\begin{array}{l}\text { Solucionar problemas de forma sistemática, experimentación, aprendizaje } \\
\text { de la experiencia pasada, aprendizaje de otros, transferencia de } \\
\text { conocimientos. }\end{array}$ \\
\hline 13. Slater y Narver (1995) & $\begin{array}{l}\text { Mentalidad emprendedora, orientación al mercado, estructura orgánica, } \\
\text { liderazgo facilitador, planificación estratégica descentralizada. }\end{array}$ \\
\hline 14. Terziovski ot al. $(2000)$ & $\begin{array}{l}\text { Modelos mentales, dominio personal, aprendizaje en equipo, idea de } \\
\text { sistemas, visión compartida. }\end{array}$ \\
\hline
\end{tabular}

Fuente: (TARÍ ET AL., 2009)

Revista Produção Online, Florianópolis, SC, v.14, n. 2, p. 720-743, abr./jun. 2014. 
Tabla 4 - Dimensiones empíricas según la literatura.

\begin{tabular}{|c|c|}
\hline Estudios & Dimensiones empiricas \\
\hline \multicolumn{2}{|c|}{ Creacion (aprondizaje organizativo) } \\
\hline 15. Goh y Richards (1997) & $\begin{array}{l}\text { Claridad de propósito y misión, liderazgo comprometido y capacitación, } \\
\text { expenmentación y recompensas, transferencia de conocimiento, trabajo en } \\
\text { grupo y resolución de problemas. }\end{array}$ \\
\hline 16. Hult y Ferell (1997) & $\begin{array}{l}\text { Orientación de equipo, orientación de sistemas, orientación de aprendizaje, } \\
\text { orientación a la memoria. }\end{array}$ \\
\hline $\begin{array}{l}\text { 17. Crossan y Hulland } \\
(2002)\end{array}$ & $\begin{array}{l}\text { Stocks de aprendizaje a nivel individual, stocks de aprendizaje en niveles } \\
\text { de grupo, stocks de aprendizaje en el nivel organizativo, flujos de } \\
\text { aprendizaje feed-forward, flujos de aprendizaje feed-back. }\end{array}$ \\
\hline 18. Jerez ot al. (2004) & $\begin{array}{l}\text { Compromiso directivo, visión del sistema, apertura y experimentación, } \\
\text { transferencia e integración del conocimiento para que una organización } \\
\text { aprenda. }\end{array}$ \\
\hline 19. Pérez et al. (2004) & $\begin{array}{l}\text { Adquisición interna de conocimiento, adquisición externa de conocimiento, } \\
\text { distribución del conocimiento, interpretación del conocimiento, memoria } \\
\text { organizativa. }\end{array}$ \\
\hline 20. Prieto y Revilla (2004) & Flujos de aprendizaje, clima de aprendizaje. \\
\hline 21. Balbastre (2001) & $\begin{array}{l}\text { Actumulación de experiencia, articulación de conocimiento y codificación } \\
\text { de conocimiento. }\end{array}$ \\
\hline 22. Calantone et al (2002) & $\begin{array}{l}\text { Compromiso con el aprendizaje, visión compartida, pensamiento abierto, } \\
\text { compartir conocimiento intraorganizacional. }\end{array}$ \\
\hline $\begin{array}{l}\text { 23. Martinez y Ruiz } \\
\text { (2003b) }\end{array}$ & Capacidad de aprendizaje, estructura organizativa, cultura organizativa. \\
\hline \multicolumn{2}{|c|}{ Transforencia y almacenamiento (conocimiento organizativo) } \\
\hline 24. Prieto y Revilla (2004) & Stocks de conocimiento \\
\hline 25. Molina et al. (2007) & $\begin{array}{l}\text { Conocimiento intemo, conocimiento de los clientes y conocimiento de los } \\
\text { proveedores. }\end{array}$ \\
\hline \multicolumn{2}{|c|}{ Aplicación y uso (organización de aprendizqje) } \\
\hline 26. Senge (1992) & $\begin{array}{l}\text { Pensamiento sistémico, dominio personal, modelos mentales, construir una } \\
\text { visión compartida y aprendizaje en equipo }\end{array}$ \\
\hline
\end{tabular}

Fuente: (TARÍ ET AL., 2009)

Revista Produção Online, Florianópolis, SC, v.14, n. 2, p. 720-743, abr./jun. 2014. 
Tabla 5 - Dimensiones propuestas de la gestión del conocimiento

\begin{tabular}{|c|c|c|}
\hline Dimensiones de la gestión del conocimiento & Estudios teóricos & Estudios empíricos \\
\hline \multicolumn{3}{|l|}{ Creación (aprendizaje organizativo) } \\
\hline Adquisición de información & $1,6,8,9,10,11$ & 19,23 \\
\hline Diseminación de la información & $6,8,9,10,11$ & 19,23 \\
\hline Interpretación compartida & $6,8,9,10,11$ & 19,23 \\
\hline \multicolumn{3}{|c|}{ Transferencia y almacenamiento (conocimiento organizativo) } \\
\hline Almacenar conocimiento & 10,11 & 17,24 \\
\hline Transferencia conocimiento en la organización & $2,5,10,11,12$ & $15,17,18,19,20,21,22,25$ \\
\hline \multicolumn{3}{|l|}{ Aplicación y uso (organización de aprendizaje) } \\
\hline Trabajo en equipo & $3,4,6,10,14$ & $15,16,26$ \\
\hline Empowement & $5,6,10,14$ & 26 \\
\hline Promover el diálogo & $5,6,7,10,12,14$ & $18,22,26$ \\
\hline $\begin{array}{l}\text { Establecer sistemas para capurar y compartir el } \\
\text { aprendizaje }\end{array}$ & $6,9,10$ & 18,26 \\
\hline $\begin{array}{l}\text { Relación entre distintos departamentos o áreas } \\
\text { funcionales }\end{array}$ & $3,6,7,10,13,14$ & $15,16,18,22,23,26$ \\
\hline Compromiso con el aprendizaje & $6,7,10,14$ & $16,22,23$ \\
\hline
\end{tabular}

Fuente: (TARÍ ET AL., 2009)

Dentro del contexto de una empresa, si definimos la gestión del conocimiento como un proceso, un enfoque de este proceso podría estar integrado básicamente, por la generación, la codificación, la transferencia y la utilización del conocimiento (WIIG, 1997).

- Generación del conocimiento: estudia los procesos de adquisición de conocimiento externo y de creación del mismo en las organizaciones, poniendo en acción los conocimientos poseídos por las personas (BUENO, 2002).

Revista Produção Online, Florianópolis, SC, v.14, n. 2, p. 720-743, abr./jun. 2014. 
- Codificación, almacenamiento o integración del conocimiento: poner al alcance de todos el conocimiento organizativo, ya sea de forma escrita o localizando a la persona que lo concentra.

- Transferencia del conocimiento: analiza los espacios de intercambio del conocimiento y los procesos técnicos o plataformas que lo hacen posible (Bueno, 2002). Esta fase puede realizarse a través de mecanismos formales $y / o$ informales de comunicación.

- Utilización del conocimiento: la aplicación del conocimiento recientemente adquirido en las actividades rutinarias de la empresa.

La generación y transferencia del conocimiento son procesos que cuenta con una mayor cantidad de conocimiento tácito. Tanto en la etapa de codificación como en la etapa de utilización, el conocimiento tácito es convertido en conocimiento explícito para la comprensión y disposición del mismo de todos los miembros de la empresa. Bueno (2002) señala que los aspectos fundamentales de la Gestión del Conocimiento son la creación y la distribución del conocimiento.

En la Tabla 6.1 y 6.2, se puede ver un resumen de los procesos de la gestión por el conocimiento por diferentes autores, en donde se muestra algunos criterios dados por diferentes autores de los procesos de gestión del conocimiento recopilados por Quintana Fundora (2006).

Tabla 6.1- Procesos de la Gestión por el Conocimiento por diferentes autores

(continua)

\begin{tabular}{|c|c|c|}
\hline AUTOR & & \\
\hline Wiig (1997) & $\begin{array}{l}\text { - Generación } \\
\text { - Adquisición } \\
\text { - Creación } \\
\text { - Uransficación, almacenamiento o integración } \\
\text { Utilización }\end{array}$ & 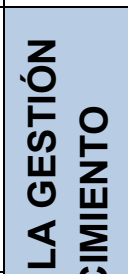 \\
\hline Hernan Gómez (1998) & $\begin{array}{l}\text { Creación } \\
\text { Desarrollo } \\
\text { Difusión }\end{array}$ &  \\
\hline Revilla y Pérez (1998) & $\begin{array}{ll}\text { - } & \text { Creación } \\
\text { - } & \text { Desarrollo } \\
\text { - } & \text { Difusión } \\
\text { - Explotación }\end{array}$ &  \\
\hline
\end{tabular}

Revista Produção Online, Florianópolis, SC, v.14, n. 2, p. 720-743, abr./jun. 2014. 
Tabla 6.1- Procesos de la Gestión por el Conocimiento por diferentes autores

(conclusão)

\begin{tabular}{|c|c|c|}
\hline AUTOR & & \\
\hline Benjamin Ditzel (2005) & 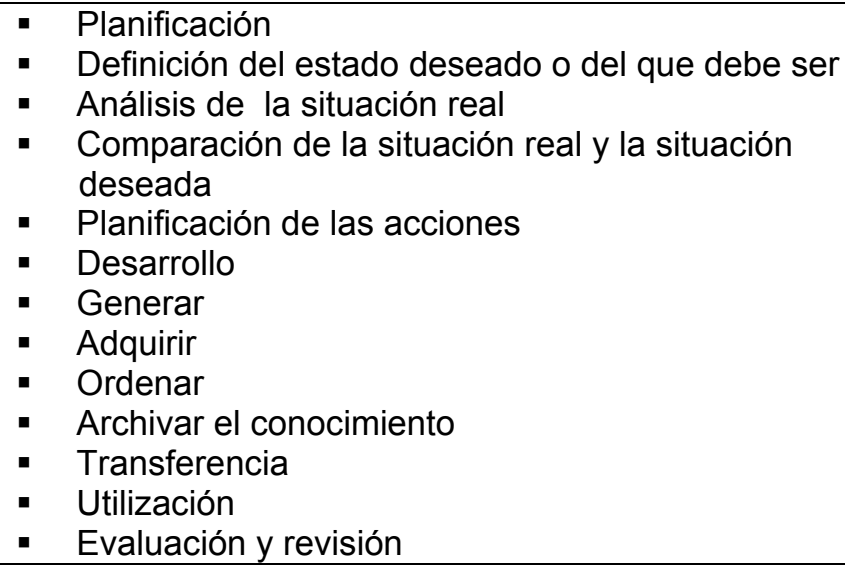 & - \\
\hline Alavi y Leiden (1999) & $\begin{array}{ll}\text { - } & \text { Creación } \\
\text { - } & \text { Compartir } \\
\end{array}$ & \\
\hline Grant (2000) & $\begin{array}{ll}\text { - } & \text { Generación } \\
\text { - } & \text { Adquisición externa } \\
\text { - } & \text { Creación interna } \\
\text { - } & \text { Aplicación } \\
\text { - } & \text { Identificación } \\
\text { - } & \text { Almación } \\
\text { - } & \text { Transfenamiento } \\
\end{array}$ & \\
\hline LLoria (2000) & $\begin{array}{ll}\text { : } & \text { Identificación } \\
\text { : } & \text { Creación } \\
\text { : } & \text { Desarrollo } \\
\text { : } & \text { Renonormación } \\
\text { : } & \text { Difusión } \\
& \text { Aplicación o utilización }\end{array}$ & \\
\hline Pavez Salazar, A (2000) & $\begin{array}{ll}\text { - } & \text { Detección } \\
\text { - } & \text { Selección } \\
\text { - } & \text { Organización } \\
\text { - } & \text { Generación } \\
\text { - } & \text { Codificación } \\
\text { - } & \text { Transferencia } \\
\text { - } & \text { Filtrado } \\
\text { - } & \text { Presentación } \\
\text { - } & \text { Utilización } \\
\end{array}$ & \\
\hline $\begin{array}{l}\text { Wensley y Verwijk- } \\
\text { O'Sullivan (2000) }\end{array}$ & $\begin{array}{ll}\text { - } & \text { Generación } \\
\text { - } & \text { Codificación } \\
\text { - } & \text { Refinamiento } \\
\text { - Transmisión }\end{array}$ & - \\
\hline
\end{tabular}

Fuente: Elaboración propia a partir de (QUINTANA, 2006)

Revista Produção Online, Florianópolis, SC, v.14, n. 2, p. 720-743, abr./jun. 2014. 
Tabla 6.2: Procesos de la Gestión por el Conocimiento por diferentes autores

\begin{tabular}{|c|c|c|}
\hline AUTOR & & \\
\hline $\begin{array}{c}\text { Alejandro A. Pavez } \\
\text { Salazar (2000) }\end{array}$ & $\begin{array}{l}\text { - } \text { Detectar y seleccionar } \\
\text { - Organizar y filtrar } \\
\text { - Presentar y usar }\end{array}$ & \multirow{13}{*}{ 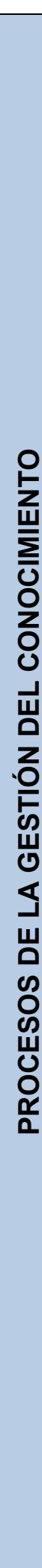 } \\
\hline Alavi y Leidner (2001) & $\begin{array}{l}\text { - } \text { Creación } \\
\text { - Almacenar Recuperar } \\
\text { - Transferencia } \\
\text { - Aplicación }\end{array}$ & \\
\hline Bhatt (2001) & $\begin{array}{l}\text { - Creación Validación } \\
\text { - Presentación } \\
\text { - Distribución } \\
\text { - Aplicación }\end{array}$ & \\
\hline $\begin{array}{l}\text { APQC citado por } \\
\text { Luan/Serban (2002) }\end{array}$ & $\begin{array}{l}\text { - Identificación } \\
\text { - Captura } \\
\text { - Transferencia }\end{array}$ & \\
\hline Alavi y Tiwana (2002) & $\begin{array}{l}\text { - } \text { Creación } \\
\text { - Codificar } \\
\text { - Aplicación }\end{array}$ & \\
\hline Lai y Chu (2002) & $\begin{array}{l}\text { - } \text { Generación } \\
\text { - } \text { Modelar Repositorio } \\
\text { - } \text { Distribución y transferencia } \\
\text { - Uso }\end{array}$ & \\
\hline Lee y Hong (2002) & $\begin{array}{ll}\text { - } & \text { Captar } \\
\text { - } & \text { Desarrollar } \\
\text { - } & \text { Compartir } \\
\text { - } & \text { Utilizar }\end{array}$ & \\
\hline Petrides/Nodine (2003) & $\begin{array}{l}\text { - } \text { Generación } \\
\text { - Almacenamiento } \\
\text { - Distribución } \\
\text { - Utilización }\end{array}$ & \\
\hline Manual ARIS (2003) & $\begin{array}{l}\text { - Adquisición } \\
\text { - Presentación } \\
\text { - Transferencia } \\
\text { - Utilización } \\
\text { - Eliminación }\end{array}$ & \\
\hline $\begin{array}{c}\text { Corrêa da Silva y Agustí- } \\
\text { Cullel (2003) }\end{array}$ & $\begin{array}{l}\text { - Generar y adquirir } \\
\text { - Almacenar y guardar } \\
\text { - Acceder- utilizar }\end{array}$ & \\
\hline $\begin{array}{c}\text { Blázquez Soriano, J.M } \\
\text { (2004) }\end{array}$ & $\begin{array}{l}\text { - } \text { Generación } \\
\text { - } \text { Captura } \\
\text { - } \text { Procesamiento } \\
\text { - Almacenamiento }\end{array}$ & \\
\hline McCann y Buckner (2004) & $\begin{array}{l}\text { - Adquirir y construir } \\
\text { - Compartir y retener } \\
\text { - Aplicar } \\
\end{array}$ & \\
\hline $\begin{array}{l}\text { Peluffo Argón, M. Beatriz } \\
\text { (2005) }\end{array}$ & $\begin{array}{l}\text { - Generación } \\
\text { - Compartición o distribución } \\
\text { - Utilización }\end{array}$ & \\
\hline
\end{tabular}

Fuente: Elaboración propia a partir de (QUINTANA, 2006)

Revista Produção Online, Florianópolis, SC, v.14, n. 2, p. 720-743, abr./jun. 2014. 
Hay que definir, por la relevancia que puede tomar, el concepto de pérdida o fugas de conocimiento, en donde nos encontramos con la extensa problemática de la pérdida del personal importante en la organización (factor que se produce con gran incidencia entre el personal de oficio, debido a la gran rotación de dicho personal o por las políticas de subcontratación de las empresas). Claramente se demuestra que el abandono de los individuos clave resulta una pérdida neta de conocimiento, limitando el grado al acceso del conocimiento y al aprendizaje para los empleados que los sustituyen al no poder contratar a un nuevo trabajador igualmente rentable. Una alta tasa de abandono rompe la continuidad en la organización y provoca un entorno social en el que los trabajadores desconfían de sus compañeros (PEREZ DE MIGUEL, 2006).

\section{HERRAMIENTAS Y TECNOLOGÍAs PARA LA GESTIÓN DEL CONOCIMIENTO}

Es evidente que para la adecuada gestión del conocimiento, hace falta una serie de herramientas y tecnologías (KIM et al., 2009; GRAY et al., 2006; SHER et al., 2004; DAVENPORT, 1997; WONG et al., 2004), que produzcan un abaratamiento y confiera una evidente fiabilidad y eficiencia en la difícil tarea de capturar el conocimiento estratégico y que genera valor para la organización. Las empresas industriales japonesas fueron pioneras en el estudio y la aplicación de su gestión, sobre todo el sector del automóvil (tabla 7), tal y como define Rivas (RIVAS et al, 2007), o con la definición de Binney (BINNEY, 2001), del espectro de la gestión

Revista Produção Online, Florianópolis, SC, v.14, n. 2, p. 720-743, abr./jun. 2014. 
Tabla 7 - Prácticas de la gestión del conocimiento en la industria del automóvil

\begin{tabular}{|c|c|c|c|}
\hline Organizaciones & $\begin{array}{l}\text { Procesos existentes } \\
\text { de Conocimiento }\end{array}$ & Origen & $\begin{array}{l}\text { Tecnologia de Intormación } \\
\text { empleada }\end{array}$ \\
\hline Nissan & Socializar el conocimiento & Necesidad de Innevar & $\begin{array}{l}\text { Correo electrónico, almacena- } \\
\text { miento de datos. }\end{array}$ \\
\hline Toyota & Conocimiento tácito & $\begin{array}{l}\text { Salir de un estatus de como- } \\
\text { didad }\end{array}$ & $\begin{array}{l}\text { Sistemas de comunicación de } \\
\text { voz }\end{array}$ \\
\hline Honda & Aprendizaje vivencial & Ventaja competitiva & $\begin{array}{l}\text { Intranets, correo electrónico, } \\
\text { comunicación de voz }\end{array}$ \\
\hline Ford & Comunidades de prictica & $\begin{array}{l}\text { Socialización del conocimiento, } \\
\text { Conocimiento explicito }\end{array}$ & $\begin{array}{l}\text { Intranet, correo electrónico, } \\
\text { almacenamiento de datos }\end{array}$ \\
\hline General Motors & Alianzas de aprendizaje & $\begin{array}{l}\text { Sobrevivir /Adquisición del } \\
\text { exterior a través de aliarzas }\end{array}$ & $\begin{array}{l}\text { Intranets, correo electrónico, } \\
\text { almacenamiento de datos }\end{array}$ \\
\hline Chrysler & $\begin{array}{l}\text { Libros de conocimiento de } \\
\text { ingeniería }\end{array}$ & Innovaciôn en productos & $\begin{array}{l}\text { Almacenamiento de datos, in- } \\
\text { tranets }\end{array}$ \\
\hline Irizar & Conocimiento Explicito & $\begin{array}{l}\text { Ventaja competitiva Evêtar du- } \\
\text { plicar la búsqueda de solución } \\
\text { a problemas }\end{array}$ & Intranet, correo electrónico \\
\hline Volve & $\begin{array}{l}\text { Socialización del conoci- } \\
\text { miento }\end{array}$ & $\begin{array}{l}\text { Ubicar las habilidades y cono- } \\
\text { cimientos del personal }\end{array}$ & $\begin{array}{l}\text { Intranet, directorios electróni- } \\
\text { cos, agentes inteligentes. }\end{array}$ \\
\hline
\end{tabular}

Fuente: (RIVAS et al., 2007).

Tabla 8 - El espectro de la GM, sus herramientas y tecnologías.

\begin{tabular}{|c|c|c|c|c|c|c|}
\hline & Transaccional & Analítica & Gestión de Bienes & Proceso & Desarrollo & $\begin{array}{c}\text { Innovación y } \\
\text { Creación }\end{array}$ \\
\hline 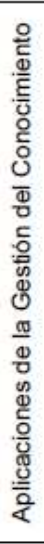 & $\begin{array}{l}\text { - Razonamiento basado en } \\
\text { el caso (RBC) } \\
\text { - Aplicaciones de ayuda en } \\
\text { el escritorio } \\
\text { - Aplicaciones de servicio } \\
\text { al cliente } \\
\text { - Aplicaciones de entrada } \\
\text { de pedidos } \\
\text { - Aplicaciones de Apoyo al } \\
\text { Agente de servicio }\end{array}$ & $\begin{array}{l}\text { - Almacenamiento de } \\
\text { datos } \\
\text { - Búsqueda de datos } \\
\text { - Inteligencia de } \\
\text { negocios } \\
\text { - Sistemas de gestión } \\
\text { de la información } \\
\text { - Sistemas de apoyo } \\
\text { a las decisiones } \\
\text { - Gestión de } \\
\text { relaciones con los } \\
\text { clientes (GRC) } \\
\text { Inteligencia } \\
\text { competitiva }\end{array}$ & $\begin{array}{l}\text { - Propiedad Intelectual } \\
\text { - Gestión de documentos } \\
\text { - Evaluación del } \\
\text { conocimiento } \\
\text { - Repositorios del } \\
\text { conocimiento } \\
\text { - Gestión del Contenido }\end{array}$ & $\begin{array}{l}\text { - TQM } \\
\text { - Benchmarking } \\
\text { - Mejores prácticas } \\
\text { - Gestión de } \\
\text { calidad } \\
\text { - Reingenieria del } \\
\text { proceso de } \\
\text { negocio } \\
\text { - Mejoramiento del } \\
\text { proceso } \\
\text { - Sistematización } \\
\text { del proceso } \\
\text { - Lecciones } \\
\text { aprendidas } \\
\text { - Metodología } \\
\text { - SEIICMM } \\
\text { ISOOXXX, SeíS } \\
\text { Sigma } \\
\end{array}$ & $\begin{array}{l}\text { - Desarrollo de } \\
\text { habilidades } \\
\text { - Competencia } \\
\text { s del } \\
\text { personal } \\
\text { - Aprendizaje } \\
\text { - Enseñanza } \\
\text { - Entrenamient } \\
\text { o }\end{array}$ & $\begin{array}{l}\text { - Comunidades } \\
\text { - Colaboración } \\
\text { - Foros de } \\
\text { discusión } \\
\text { - Redes } \\
\text { - Equipos virtuales } \\
\text { - Investigación y } \\
\text { desarrollo } \\
\text { - Equipos muiti- } \\
\text { - disciplinarios }\end{array}$ \\
\hline  & $\begin{array}{l}\text { - Sistemas expertos } \\
\text { - Tecnologias cognitivas } \\
\text { - Redes semánticas } \\
\text { - Sistemas de expertos } \\
\text { basados en reglas } \\
\text { - Redes de probabilidades } \\
\text { - Inducción a las reglas- } \\
\text { Árboles de decisiones } \\
\text { - Sistemas de información } \\
\text { geoespacial }\end{array}$ & $\begin{array}{l}\text { - Agentes inteligentes } \\
\text { - Expertos en internet } \\
\text { - DBMS de objetos y } \\
\text { relaciones } \\
\text { - Cómputos neurales } \\
\text { - Tecnologías } \\
\text { impuestas } \\
\text { - Análisis de datos y } \\
\text { herramientas para } \\
\text { los informes }\end{array}$ & $\begin{array}{l}\text { - Herramientas de } \\
\text { gestión de documentos } \\
\text { - Motores de búsqueda } \\
\text { - Mapas de conocimiento } \\
\text { - Sistemas de biblioteca }\end{array}$ & $\begin{array}{l}\text { - Gestión del flujo } \\
\text { de trabajo } \\
\text { - Herramientas de } \\
\text { los modelos del } \\
\text { proceso }\end{array}$ & $\begin{array}{l}\text { - Entrenamient } \\
\text { o basado en } \\
\text { los } \\
\text { computadore } \\
\text { s } \\
\text { - Entrenamient } \\
\text { o en linea }\end{array}$ & $\begin{array}{l}\text { - Groupware- } \\
\text { trabajo conjunto } \\
\text { - Correo electrónico } \\
\text { - Salas de chat } \\
\text { - Video } \\
\text { conferencias } \\
\text { - Motores de } \\
\text { búsqueda } \\
\text { - Correo de voz } \\
\text { - Carteleras } \\
\text { - Tecnologias } \\
\text { impuestas } \\
\text { - Tecnologias de } \\
\text { simulacro }\end{array}$ \\
\hline
\end{tabular}

Fuente: (BINNEY, 2001)

Son numerosos los autores, que indican como valores relevantes, el conocer

lo que queremos gestionar (el conocimiento), mediante las auditorias de Revista Produção Online, Florianópolis, SC, v.14, n. 2, p. 720-743, abr.jjun. 2014. 
mantenimiento, y clarificar como se distribuye dicha información-conocimiento en el seno de la organización, mediante herramientas sencillas, visuales e intuitivas, como pueden ser los mapas de información o los mapas de conocimiento.

\subsection{Los mapas de conocimiento}

Un primer paso a dar, por evidente que parezca, es la identificación de los conocimientos que residen en el seno de la misma así como de sus características o elementos identificativos.

Un mapa del conocimiento es una herramienta para la localización del conocimiento dentro de una organización. Es similar a un mapa de información pero orientado a conocimiento en lugar de información. Puede tener una representación pictórica en forma de una red de conocimiento (GIL et al., 2008).

Algunas de las razones para elaborar el mapa de conocimiento organizacional (PEREZ, 2005), pueden ser definidas por las siguientes:

- Para encontrar fuentes claves y restricciones en la creación de conocimiento y en sus flujos.

- Para animar la reutilización y prevenir la reinvención, identificando prácticas repetitivas, ahorrando tiempo de búsqueda y reduciendo los costes de adquisición.

- Para identificar las islas de experiencia y sugerir modos de construir puentes para incrementar la compartición de conocimiento (GOH et al., 2009).

- Para descubrir las comunidades eficaces y emergentes de práctica donde sucede el aprendizaje.

- Para mejorar los tiempos de respuesta al cliente, la toma de decisiones y la solución de problemas, proporcionando acceso a la información requerida.

- Para destacar oportunidades para el aprendizaje y distribución de conocimiento distinguiendo un significado único de "conocimiento" dentro de la organización. En el ámbito organizacional esto permite informar sobre el desarrollo de una estrategia de conocimiento.

- Para desarrollar una arquitectura de conocimiento o una memoria corporativa.

El mapa del conocimiento organizacional permite el diagnóstico de cada problema en su contexto particular, lo que hace más fácil identificar las partes de la Revista Produção Online, Florianópolis, SC, v.14, n. 2, p. 720-743, abr./jun. 2014. 
organización afectadas y que pueden ser involucradas en la búsqueda de una solución. En el se recogerán todos los conocimientos detectados, así como una descripción de su contenido y sus principales características.

Quintana (QUINTANA, 2006) afirma que un mapa de conocimiento es un mapa actualizado que nos indica cuál es el conocimiento existente y dónde se encuentra, pero que no contiene al mismo conocimiento, solo la referencia de dónde encontrarlo. El desarrollo de un mapa de conocimiento supone localizar el conocimiento importante para la organización y, posteriormente, publicar listas o representaciones que muestren donde encontrarlo.

Según d'Alòs-Moner (d'Alos-Moner, 2003) los mapas del conocimiento permiten tener una visión gráfica de cuál es la situación de la organización en relación con su conocimiento, entendido como parte de su capital intelectual.

Para Bueno (2003) el mapa de conocimiento es un conjunto de información capaz de ser fácilmente asimilable, es decir, convertirse en conocimiento. Permite encontrar eficientemente información relevante para la toma de decisiones y la resolución de problemas.

El mapa puede hacer referencia a personas, instituciones, documentos en cualquier soporte y bases de datos propias o externas. Un mapa de conocimiento es la exposición visual de información capturada mediante texto, gráficos, modelos o números, así como de las relaciones existentes dentro de dicha información (VAIL, 1999).

Para Seemann et al. (1997), los mapas de conocimiento muestran dónde encontrar fuentes importantes de conocimiento en la organización, apuntando a repositorios de documentos importantes o a personas expertas en alguna materia. De otro lado, el uso de repositorios de documentos es más beneficioso si se construyen siguiendo los principios de los mapas de conocimiento.

Un repositorio de documentos es un "almacén" de documentos que contienen conocimiento. Según Grover et al. (2001), los repositorios normalmente contienen un tipo específico de conocimiento para una función o proceso de negocio concreto. El objetivo es capturar el conocimiento para que posteriormente muchos otros miembros de la compañía puedan tener acceso a ese conocimiento. En la tabla 9 se describen las principales características de un mapa del conocimiento. 
Tabla 9 - Características fundamentales de los mapas de conocimiento

\begin{tabular}{|c|c|c|}
\hline \multicolumn{3}{|c|}{ CARACTERÍSTICAS MAPAS DEL CONOCIMIENTO } \\
\hline ATRIBUTOS & $\begin{array}{l}\text { FUENTES DEL MAPA } \\
\text { DEL CONOCIMIENTO }\end{array}$ & $\begin{array}{l}\text { UTILIDADES DEL MAPA DEL } \\
\text { CONOCIMIENTO }\end{array}$ \\
\hline $\begin{array}{l}\text { Constituye la } \\
\text { recopilación de los } \\
\text { conocimientos de los } \\
\text { que se dispone en } \\
\text { una unidad / empresa. } \\
\text { Enumeración de } \\
\text { conocimiento } \\
\text { explicitado y } \\
\text { documentado, y } \\
\text { también conocimiento } \\
\text { tácito que tienen las } \\
\text { personas relevantes. } \\
\text { Conocimiento } \\
\text { priorizado y agrupado. } \\
\text { El mapa nos indica, } \\
\text { además, cómo llegar } \\
\text { a este conocimiento } \\
\text { relevante: qué } \\
\text { personas lo tienen, en } \\
\text { qué soporte se } \\
\text { encuentra, etc. } \\
\text { Permite identificar las } \\
\text { lagunas de } \\
\text { conocimiento. } \\
\text { El mapa del } \\
\text { conocimiento } \\
\text { pretende ser la } \\
\text { herramienta de diseño } \\
\text { y mantenimiento del } \\
\text { programa de gestión } \\
\text { por el conocimiento. }\end{array}$ & $\begin{array}{l}\text { Estructuradas, como } \\
\text { por ejemplo datos de } \\
\text { una base de datos } \\
\text { interna o informes } \\
\text { procedentes de } \\
\text { proveedores externos. } \\
\text { Información } \\
\text { desestructurada en } \\
\text { diferentes } \\
\text { documentos y tipos de } \\
\text { soporte. } \\
\text { Conocimiento tácito } \\
\text { localizado en la mente } \\
\text { de un experto. }\end{array}$ & $\begin{array}{l}\text { Facilita la concentración de } \\
\text { recursos en los procesos de } \\
\text { creación del conocimiento. } \\
\text { Evita que las personas se } \\
\text { dediquen a crear conocimientos } \\
\text { que ya existen. } \\
\text { Permite localizar la mejor fuente / } \\
\text { experto para conseguir un } \\
\text { conocimiento. } \\
\text { Identificar necesidades de } \\
\text { conocimiento, y el conocimiento } \\
\text { que hay que desaprender. } \\
\text { Identificación de las áreas y } \\
\text { procesos donde la implantación } \\
\text { de una iniciativa de gestión del } \\
\text { conocimiento proporcionará más } \\
\text { valor a la organización. } \\
\text { Es la base para el diagnóstico de } \\
\text { la gestión del conocimiento } \\
\text { identificado y la búsqueda de } \\
\text { acciones de mejora. } \\
\text { Aplicación inmediata a otros } \\
\text { procesos: de gestión de } \\
\text { información, intranet, gestión de } \\
\text { calidad, etc. } \\
\text { Indica dónde pueden establecerse } \\
\text { las comunidades y centros de } \\
\text { interés o de práctica. } \\
\text { Formalización y organización de } \\
\text { todos los inventarios de } \\
\text { conocimiento. } \\
\text { Percepción de las relaciones } \\
\text { entre los conocimientos. } \\
\text { inventario del conocimiento. } \\
\text { Promoción de la } \\
\text { socialización/externalización } \\
\text { conectando a los expertos con los } \\
\text { exploradores del conocimiento. }\end{array}$ \\
\hline
\end{tabular}

Fuente: Elaboración propia a partir de (SEEMAN et al. 1997)

En resumen los resultados de un mapa de conocimiento deben ser:

- La generación de conocimiento.

- La presentación

- La transferencia e intercambio del conocimiento.

Revista Produção Online, Florianópolis, SC, v.14, n. 2, p. 720-743, abr./jun. 2014. 
La integración de este conocimiento en la organización y un medio para llegar hacia la "organización que aprende".

A modo de ejemplo se puede apreciar en la figura 1, un mapa de conocimiento que nos muestra de manera gráfica las actividades que se requieren realizar en un proceso que se está describiendo, el responsable de dicho proceso, el conocimiento requerido, el conocimiento creando, el conocimiento faltante, los usuarios, el conocimiento proporcionado, sus usos y como fluye entre los poseedores del conocimiento y los destinatarios.

Figura 1 - Ejemplo de mapa de conocimiento de una sesión tutorial.

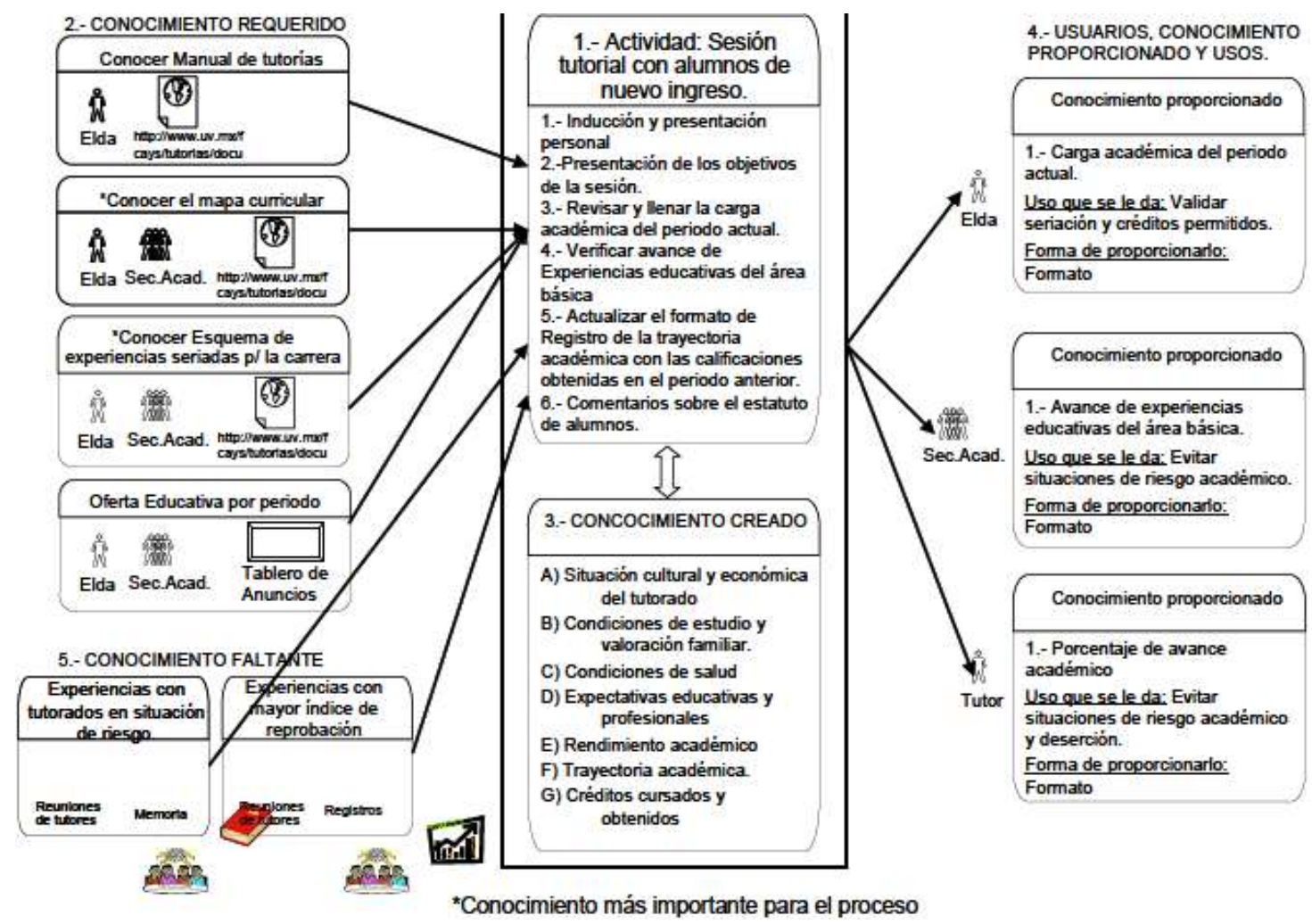

Fuente: (GIL et al., 2008)

Hay que tener en cuenta en la construcción de un mapa de conocimiento se deben de realizar 4 actividades (HANSEN, 2004):

1. Dibujar todos los elementos importantes de la estructura organizacional: Seleccionar un área de la organización para empezar y, a partir de ésta, comenzar a dibujar las unidades organizacionales, documentos, sistemas informáticos, o recurso humano. Este último elemento, se pueden indicar

Revista Produção Online, Florianópolis, SC, v.14, n. 2, p. 720-743, abr./jun. 2014. 
características adicionales como sus roles específicos e importancia. Se pueden utilizar imágenes que representen los informes escritos y utilizar sus abreviaciones formales para distinguirlos. La misma estrategia se puede utilizar para describir los sistemas informáticos. Lo importantes es que sean comprendidos por los participantes que ayudarán posteriormente a analizar el mapa en caso de que sea necesaria su validación.

2. Describir todos los flujos de conocimiento: Se especifica el flujo entre dos o más personas o elementos de conocimiento y se indica lo que representa ese flujo. Se pueden utilizar los diferentes para representar los flujos dependiendo el nivel del mismo.

3. Proporcionar el contexto para los flujos de conocimiento: Una vez que el mapa es analizado y validad por la organización, se identifican características adicionales para ser añadidas al mapa, y se identifican cuáles son los flujos problemáticos y cuáles se han omitido. Se puede utilizar otro color para destacar las áreas problemáticas, y se marcan con un signo de exclamación grande. Finalmente, este paso también puede usarse para indicar sobre el mapa donde se pueden generar nuevas ideas e iniciativas señalándolas con la imagen de un foco.

4. Analizar los problemas identificados para entender sus orígenes y causas: Esto se complementa en el mapa con una lista de áreas de mejoras. El mapa permite el diagnóstico de cada problema con su contexto particular en lo que concierne a la estructura y el proceso, que hace más fácil identifica qué partes de la organización están afectadas y que pueden ser involucradas en la búsqueda de una solución.

\subsection{La auditoría del conocimiento}

La auditoría del conocimiento es el primer paso que se debe llevar a cabo en los proyectos de gestión de conocimiento (GC).

En la literatura revisada se ha encontrado un uso de ontologías como formalismo para representar el conocimiento como apoyo a los procesos de Auditoría del Conocimiento en las organizaciones. La necesidad de aplicar 
ontologías en el modelado de procesos es tema de interés (ROJAS et al., 2009). El modelado de procesos describe el dominio de la aplicación en términos de un sistema formado por un conjunto de elementos relacionados: objetivo, procesos, actividades (flujo de trabajo), objetos, actores, estructura organizacional, reglas de negocio y eventos. En ocasiones, el modelar cada uno de estos elementos no se apoya de un método claro que permita hacerlo. En este sentido, una ontología podría contribuir a lograr tal claridad conceptual.

Las ontologías ayudan también, a los grupos de modelado de procesos a establecer, diferenciar y relacionar objetivos, procesos, actividades, recursos, reglas, actores, tecnologías y eventos que caracterizan a los sistemas de negocios. Asimismo, facilita la comunicación entre los actores que participan en el desarrollo de software, al proporcionar una definición única y consensual de los conceptos del dominio de la aplicación.

Los resultados obtenidos de una auditoria del conocimiento, son los requisitos para desarrollar adecuadamente un proyecto de gestión del conocimiento (PANIAGUA, 2007).

En la tabla 10 se pueden observar las características fundamentales que debería cumplir una auditoría del conocimiento. 
Tabla 10 - Características fundamentales de la auditoría del conocimiento.

\begin{tabular}{|c|c|c|c|}
\hline \multicolumn{4}{|c|}{ CARACTERÍSTICAS FUNDAMENTALES DE LA AUDITORÍA DEL CONOCIMIENTO } \\
\hline $\begin{array}{l}\text { Los objetivos que } \\
\text { persigue la auditoria } \\
\text { del conocimiento } \\
\text { (Debenham, 1994): }\end{array}$ & $\begin{array}{l}\text { Resultados que } \\
\text { debe incluir un } \\
\text { reporte de } \\
\text { auditoría: }\end{array}$ & $\begin{array}{c}\text { Debe dar } \\
\text { respuesta a las } \\
\text { siguientes } \\
\text { preguntas } \\
\text { (Pérez, 2006): }\end{array}$ & Métodos de análisis \\
\hline $\begin{array}{l}\text { - Obtener una visión } \\
\text { amplia y } \\
\text { estructurada de } \\
\text { conocimiento } \\
\text { contenido en una } \\
\text { determinada } \\
\text { sección de una } \\
\text { organización. } \\
\text { - Identificar fuentes } \\
\text { de conocimiento. } \\
\text { - Caracterizar } \\
\text { cualitativa y } \\
\text { cuantitativamente el } \\
\text { conocimiento } \\
\text { ubicado en las } \\
\text { fuentes. }\end{array}$ &  & 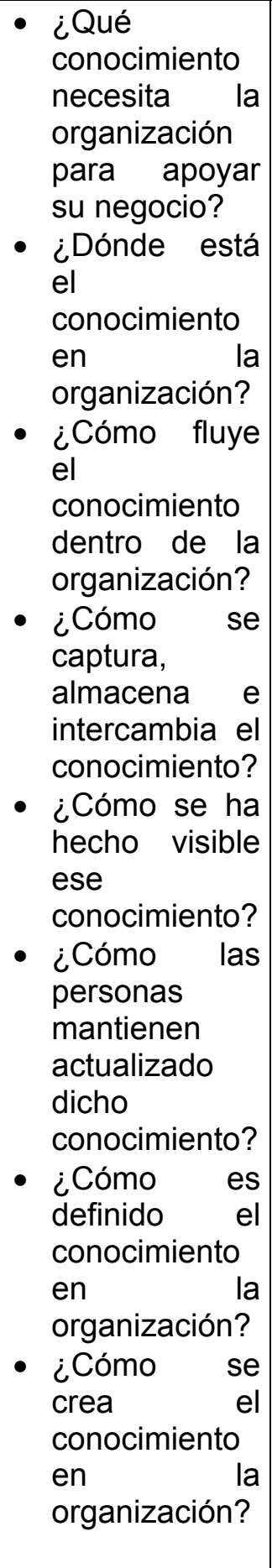 & 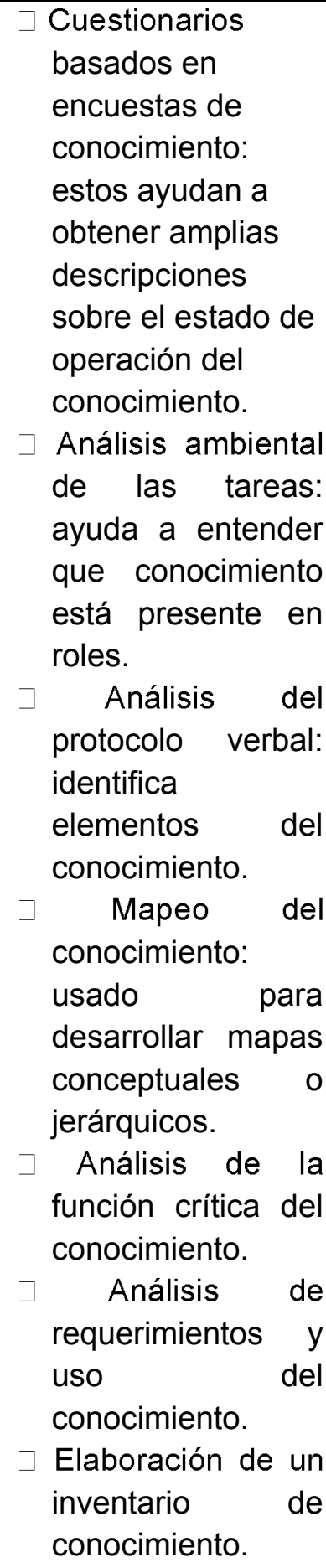 \\
\hline
\end{tabular}

Fuente: elaboración propia a partir de varios autores. 
Otros autores (LIEBOWITZ, 2000) describen otros métodos de análisis que pueden ser utilizados dentro de las auditorias:

1. Identificar el conocimiento que actualmente existe en el área a analizar.

Consiste en determinar fuentes, flujos y restricciones en el área, y localizar el conocimiento tácito e explícito y construir un mapa de conocimiento y flujo de conocimiento.

2. Identificar el conocimiento perdido en el área.

3. Proporcionar recomendaciones en la auditoria del conocimiento con respecto al estatus y a posibles mejoras para las actividades de gestión de conocimiento.

Con la auditoría se identifican oportunidades y ayuda a ubicar y evaluar las fuentes donde se encuentra almacenado el conocimiento, las actividades que transforman el conocimiento, y los factores que intervienen en estas actividades, y por ultimo permite establecer patrones de solución (PEREZ, 2007). Además ayuda a obtener los requerimientos para el diseño de un sistema eficiente de gestión de conocimiento, con la exploración de las primeras actividades a realizar en la cadena del conocimiento (HOLSAPPLE et al., 2004).

\section{CONCLUSIONES}

La generación y transferencia del conocimiento son procesos de vital importancia en la ingeniería del mantenimiento industrial, donde tradicionalmente se desarrollan los trabajos en base a una mayor cantidad de conocimiento tácito basado en la experiencia durante años de los operarios, y es preciso definir los procesos y dimensiones asociados a los procesos de gestión del conocimiento en un área tan compleja como son las organizaciones de mantenimiento de las empresas industriales. Tanto en la etapa de codificación como en la etapa de utilización, el conocimiento tácito es convertido en conocimiento explícito para la comprensión y disposición del mismo de todos los miembros de la empresa.

Se han revisado en este artículo, cuales son las taxonomías de modelos de gestión del conocimiento en donde se pueden encontrar puntos en común que permiten resumirlas y reagruparlas para poder homogenizar los criterios en áreas donde el estudio y desarrollo de la gestión del conocimiento han tenido un 
desenvolvimiento importante, y su extrapolación hacia las organizaciones de mantenimiento industrial para la adecuada gestión de su conocimiento estratégico que influye directamente en la operatividad de la empresa.

Así mismo para la adecuada gestión del conocimiento, hace falta una serie de herramientas y tecnologías, que produzcan un abaratamiento y confiera una evidente fiabilidad y eficiencia en la difícil tarea de capturar el conocimiento estratégico y que genera valor para la organización. Las empresas industriales japonesas fueron pioneras en el estudio y la aplicación de su gestión, sobre todo en el sector del automóvil, extrapolándose con posterioridad a otras actividades.

\section{AGRADECIMIENTOS}

Los autores del presente artículo quieren mostrar su agradecimiento al editor y los revisores anónimos por las indicaciones y propuestas que han ayudado a mejorar la presentación del presente estudio.

\section{REFERENCIAS}

BARRAGÁN, A. Aproximación a una taxonomía de modelos de gestión del conocimiento. Intangible Capital, v. 5, n. 1, p. 65-101, 2009.

http://dx.doi.org/10.3926/ic.2009.v5n1.p65-101

BINNEY, D. El espectro de la gestión del conocimiento: el entendimiento del panorama de la GC. Diario de la Gestión del conocimiento, vol. 5, no. 1, p. 33-42, 2001.

BUENO, E. Enfoques principales y tendencias en Dirección del conocimiento. Knowledge Management, 2003.

Bueno, E. La sociedad del conocimiento: un nuevo espacio de aprendizaje de las personas y organizaciones, en La Sociedad del Conocimiento, Monografía de la Revista Valenciana de Estudios Autonómicos, Presidencia de la Generalitat Valenciana, Valencia, 2002.

BUENO, E. Gestión del conocimiento y capital intelectual' Experiencias en Espa-a, Comunidad de Madrid-II.I Euroforum Escorial Madrid, 1999.

D'ALÒS-MONER, A. El profesional de la información, v. 12, n. 4, julio/agosto, 2003.

Davenport $\mathrm{H}$. Ten principles of knowledge management and four case studies.

Knowledge and Process Management, v. 4, n. 3, p. 187-208, 1997. 
http://dx.doi.org/10.1002/(SICI)1099-1441(199709)4:3<187::AID-KPM99>3.3.CO;2-1

DEBENHAM, J; CLARK, J. The knowledge audit. Robotics and computerintegrated manufacturing, v. 11, n. 3, p. 201-211, 1994.

http://dx.doi.org/10.1016/0736-5845(94)90035-3

GIL, M; PÉREZ, A; LÓPEZ, G; La auditoría del conocimiento como etapa previa a la gestión del conocimiento en una institución educativa Mexicana. CONGRESO INTERNACIONAL DE ANÁLISIS ORGANIZACIONAL, 6.,2008. Annals...Nuevo Vallarta, México, Noviembre de 2008.

GRAY PH, MEISTER DB. Knowledge sourcing methods. Information Management, v. 43, n. 2, p. 142-156, 2006. http://dx.doi.org/10.1016/j.im.2005.03.002

GROVER, V.; DAVENPORT, T.H.General perspectives on knowledge management: fostering a research agenda.Journal of Management Information Systems, v. 18, n.1, 2001.

HANSEN, B; KAUTZ, K. Knowledge zapping: a technique for identifying knowledge flows in software organisations. EuroSPI. 2004. pp. 126-137, 2004.

HEDLUD, O. A model of knowledge management and the $\mathrm{N}$-form corporation. Strategic Management Joumal, v. 15, p. 73-90, 1994.

http://dx.doi.org/10.1002/smj.4250151006

HOLSAPPLE C, JONES K. Exploring primary activities in the knowledge chain. Knowledge and Process Management, v. 11, n. 3, 155-174, 2004. http://dx.doi.org/10.1002/kpm.200

Kim H, BRESLIN JG, DECKER S. (2009). Personal knowledge management for knowledge workers using social semantic technologies. International Journal Intelligent Information and Database Systems, v. 3, n. 1, p. 28-43, 2009. http://dx.doi.org/10.1504/IJIIDS.2009.023036

LIEBOWITZ, JAY ET AL.The knowledge audit, knowledge and process.

Management, v. 7, n.1, p 3-10, 2000.

http://dx.doi.org/10.1002/(SICI)1099-1441(200001/03)7:1<3::AID-KPM72>3.0.CO;2-0

NONAKA, I.; TAKEUCHI, $\mathrm{H}$. The knowledge-creating company: how japanese companies create the dynamics of innovation. Oxford University Press, New York, 1995. 
ORDOÑES DE PABLOS, P. Gestión del conocimiento y medición del capital intelectual en la empresa internacional. Proyecto de Doctorado, Universidad de Oviedo, 1999.

ORDOÑEZ DE PABLOS, P. Capital intelectual, gestión del conocimiento y sistemas de gestión de recursos humanos: Influencia sobre los resultados organizativos. Tesis Doctoral, Universidad de Oviedo, 2001.

PANIAGUA, E. La Gestión tecnológica del conocimiento. Murcia: Universidad de Murcia, Servicio de Publicaciones, 2007.

PÉREZ DE MIGUEL, A. Hacia el índice de conocimiento roto: introducción a los coeficientes de rotura de conocimiento: documentos de trabajo "nuevas tendencias en dirección de empresas", 2006.

POLANYI, M. The tacit dimension. London: Routledge \& Kegan Paul, 1966.

QUINTANA FUNDORA, Y .Gestión por el conocimiento en la carrera de Ingeniería Industrial. Administración de operaciones. Tesis en opción al título de Master en Ciencias. Facultad de Industrial Economía. Universidad de Matanzas. Cuba, 2006.

RIVAS, L; FLORES, B. La gestión de conocimiento en la industria automovilistica. Estudios Gerenciales. Colômbia, v. 23, n. 102, p. 83-100, enero/marzo 2007.

ROJAS, C. M., MONTILVA, C. J., BARRIOS, A. J. Revista Colombiana de Tecnologías Avanzadas, v. 1, n. 13, p. 72-80, 2009.

SEEMANN, P. Y COHEN, D. The geography of knowledge: from knowledge maps to the knowledge atlas. Knowledge and Process Management, v. 4, n.4, 1997.

SHER JP, LEE CV. Information technology as a facilitator for enhancing dynamic capabilities through knowledge management. Information Management, v.41, n.8, p. 933-945, 2004. http://dx.doi.org/10.1016/j.im.2003.06.004

TARI, J; GARCIA, M. Dimensiones de la gestión del conocimiento y la gestión de la calidad: una revisión de la literatura. Investigaciones Europeas de Dirección y Economía de la Empresa, v. 15, n. 3, p. 135-148, 2009.

http://dx.doi.org/10.1016/S1135-2523(12)60105-1 
TIEMESSEN, L. et al. Knowledge management in intemational joint ventures, en BEAMISH.P. W. y KILLING, J. P.: Cooperative strategies. North American perspectives. The Cooperative Strategies Series. The New Lexington Press, 1997.

VAIL, E. F. Mapping Organizational Knowledge: Bridging the business-IT communication gap. Knowledge Management Review, v. 2, n. 8, 1999.

VENTURA, J. Análisis dinámico de la estrategia empresarial: un ensayo interdisciplinar. Servicio de publicaciones. Universidad de Oviedo, 1996.

WERNERFELT, B. A resource based view ofthe firm. Strategic Management Journal, Vol.5, pp. 171-180, 1984. http://dx.doi.org/10.1002/smj.4250050207

Wiig, K.M. Integrating intellectual capital and knowledge management. Long Range Planning, v. 30, n. 3, Junio 1997.

WONG KY, ASPINWALL E. Knowledge management implementation frameworks: a review. Knowledge and Process Management, v. 11, n. 2, p. 93-104, 2004.

http://dx.doi.org/10.1002/kpm.193



Artigo recebido em 28/04/2013 e aceito para publicação em 15/05/2014

DOI: http://dx.doi.org/10.14488/1676-1901.v14i2.1577 